\title{
Corpus callosum morphology in first-episode and chronic schizophrenia: combined magnetic resonance and diffusion tensor imaging study of Chinese Singaporean patients ${ }^{\dagger}$
}

Simon L. Collinson, Swu Chyi Gan, Puay San Woon, Carissa Kuswanto, Min Yi Sum, Guo Liang Yang, Ji Min Lui, Yih Yian Sitoh, Wieslaw L. Nowinski and Kang Sim

\section{Background}

Abnormalities in the corpus callosum have been reported in influence of inter-individual differences and illness characteristics remains to be fully elucidated.

\section{Aims}

To examine the influence of individual and illness characteristics on the corpus callosum in Chinese singaporean patients with schizophrenia.

\section{Method}

Using magnetic resonance and diffusion tensor imaging, mean investigated in 120 chinese singaporean patients (52 with chronic and 68 with first-episode schizophrenia) and compared with data from 75 matched healthy controls. patients with schizophrenia for over 30 years but the corpus callosum area, volume and fractional anisotropy were

\section{Results}

Both area and volume were significantly reduced in patients relative to controls but no significant differences in corpus callosum existed between genders in either patients or controls. Differences in area and volume of the corpus callosum were greatest in patients whose condition was chronic relative to patients with a first episode and controls Anterior callosum in patients, regardless of chronicity, was no different to that of controls.

\section{Conclusions}

Morphological abnormalities in the corpus callosum may increase with illness progression.

\section{Declaration of interest} None.

indices of corpus callosum structure and white matter integrity using magnetic resonance imaging (MRI) and diffusion tensor imaging (DTI) measures.

\section{Method} and whether illness characteristics (age at onset, duratio illness) further alter the corpus callosum in people with schizophrenia remains to be fully answered. Despite a large number of investigations there are few systematic findings relating to sexual dimorphism of the corpus callosum in schizophrenia and few studies have considered individual and interactive effects of age and gender. The potential influence of illness chronicity also remains to be elucidated. Several cross-sectional magnetic resonance studies have shown no effect of age at onset, duration of untreated psychosis or illness duration on corpus callosum indices, ${ }^{2,3}$ whereas others have shown decreases in corpus callosum size with length of illness ${ }^{4}$ or reductions in callosal subsegments that vary between first-episode and established schizophrenia. ${ }^{5}$ Longitudinal studies offer the best solution ${ }^{6,7}$ and have demonstrated longitudinal changes in subsegments of the corpus callosum pointing to an abnormality in the developmental trajectory but the logistic difficulties of prospective and longitudinal studies and their focus on younger patients presently limit the conclusions that can be drawn about age- and genderrelated morphology in more chronic schizophrenia. In order to address the effect of inter-individual and illness characteristics on corpus callosum morphology and microstructural integrity in both first-episode and chronic schizophrenia, we examined

`See editorial, pp. 9-11, this issue. 
(GAF) scale. Handedness was determined with the administration of the Modified Edinburgh Questionnaire. ${ }^{12}$

Written, informed consent was obtained from all participants after a thorough explanation of the study procedures. The institutional review boards of the Institute of Mental Health and the National Neuroscience Institute approved the study protocol.

\section{Image acquisition}

Magnetic resonance imaging was performed with a 3-Tesla whole body MRI scanner (GyroscanAchieva, Philips Medical Systems, Eindhoven, The Netherlands). A regular quality-control procedure ensured the stability of a high signal to noise ratio. Whole brain volumetric scans were then acquired with a high resolution, $T_{1}$-weighted turbo-field echo sequence (repetition time $(\mathrm{TR})=8.4 \mathrm{~s}$; echo time $(\mathrm{TE})=3.8 \mathrm{~ms}$; inversion time $(\mathrm{TI})=3000 \mathrm{~ms}$; flip angle, $8^{\circ}$; field of view $(\mathrm{FOV})=230 \mathrm{~mm}^{2}$; acquisition matrix $256 \times 256$ ) that produced a total of 180 contiguous, $0.9 \mathrm{~mm}$ thick axial slices with no gaps.

Diffusion-weighted images were obtained in the same session using a single-shot echo-planar sequence ( $\mathrm{TR}=3725 \mathrm{~ms}$; $\mathrm{TE}=56 \mathrm{~ms}$; flip angle, $90^{\circ} ; b$-factor, $800 \mathrm{~s} / \mathrm{mm}^{-2}$ ) in 15 nonparallel directions with the baseline image being acquired without diffusion weighting. Each volume comprised of 42 axial $3.0 \mathrm{~mm}$ thick slices with no gap $\left(\mathrm{FOV}=230 \mathrm{~mm}^{2}\right.$; acquisition matrix $256 \times 256$ after conversion). A total of three volumes were obtained to improve signal-to-noise ratio of the scans. Structural and diffusion tensor images were acquired sequentially in one single scan time with no position change.

\section{Image processing}

Structural images were converted from the original DICOM format into the Analyze file format (v7.5). The midsagittal plane was analysed according to the previously validated method that was developed in-house and detailed in Nowinski et al. ${ }^{13}$ The method is based on the Kullback-Leibler's measure quantifying the difference between two intensity distributions. ${ }^{13}$ The magnetisation prepared rapid gradient echo (MP-RAGE) scans were processed to identify the landmarks including the anterior commissure, posterior commissure, brain extents in the left, right, anterior, posterior, dorsal and ventral directions, and the superior midway and inferior midway landmarks corresponding to the top of the corpus callosum and the bottom of the orbitofrontal cortex respectively. FreeSurfer version 4.0.5 software package (Athinoula A. Martinos Center for Biomedical Imaging, Massachusetts General Hospital, Harvard University, http://surfer.nmr.mgh. harvard.edu/) run on Linux OS (CentOs 4) was used to reformat each brain volume into a $1 \mathrm{~mm}^{3}$ isovoxels volume, and delineated into specific brain structures and automatically identify the lateral boundary of the corpus callosum. This automated method has been shown to be statistically indistinguishable from manual raters ${ }^{14}$ and reduces random errors, rater error and inter-participant variability typical of manual techniques. The corpus callosum was arbitrarily divided into five predetermined segments applied by FreeSurfer, with each section representing a fifth of the total area/volume that broadly corresponds to functional subdivisions, namely: anterior, mid-anterior, central, mid-posterior and posterior portions (Fig. 1).

Total volume of the corpus callosum, and its lateral extent, was defined by measuring a multislice three-dimensional slab of voxels of white matter pathways that run horizontally from the midsagittal plane and laterally to where pathways change direction (i.e. where they extend into the corona radiata) bilaterally. To obtain the corpus callosum area measures, the number of pixels in each corpus callosum subregion (as above) was counted on the midsagittal slice only, as each pixel is equivalent to $1 \mathrm{~mm}^{2}$.
The total callosal area was computed by adding the areas for all five corpus callosum segments.

Fractional anisotropy maps were acquired from the DTI images from the software DTI Studio, ${ }^{15}$ and were then co-registered automatically to the MP-RAGE images using a mutual-information cost function and a 12-parameter affine transformation. Eddy current correction was performed prior to registration. As the DTI images are co-registered to the participant's structural images, fractional anisotropy images were also automatically delineated for the separate brain structures using the same delineation parameters in the structural images. From this delineation, the five segments of the corpus callosum were extracted and individually compared according to the boundary determined by the volume contained within the corpus callosum volume segmentation.

The test-retest (intra-rater) reliability of the measurement technique of the corpus callosum indices (area, volume, fractional anisotropy) were assessed by repeated measurement of eight randomly selected participants (four from controls and four from patients) over a minimum interval of 2 weeks. On the basis of a two-factor random effects model for intraclass correlation coefficient calculation, alpha values were all greater than 0.90 for all the corpus callosum parameters. Interrater reliability evaluation performed on a separate subset of eight participants (four from controls and four from patients) revealed alpha values of greater than 0.90 for the corpus callosum indices.

\section{Statistical analyses}

We used IBM SPSS (version 20) for Mac for data analysis. Demographic and clinical variables were investigated with the use of one-way ANOVA corrected for multiple comparisons (Bonferroni correction). The corpus callosum parameters were then subjected to repeated measures analysis of covariance (ANCOVA) using diagnosis (first-episode patients, chronic patients, controls) as between-group factor and gender (males $v$. females) and corpus callosum parameters (corpus callosum area, volume and fractional anisotropy measures) as within-participant factors and with age, years of education, intracranial volume and daily chlorpromazine equivalent dose as covariates. Post hoc comparisons with Bonferroni corrections were performed for significant main effects. Correlations were performed to assess the relationship between the corpus callosum measures (area, volume, fractional anisotropy) and their relationship with the demographic and clinical variables.

\section{Results}

\section{Participant characteristics}

Clinical and demographic details of the participants are listed in Table 1. There were eight left-handed and one ambidextrous patient and seven left-handed controls. Out of the original 120 patients and 75 controls recruited for the study, data from 113

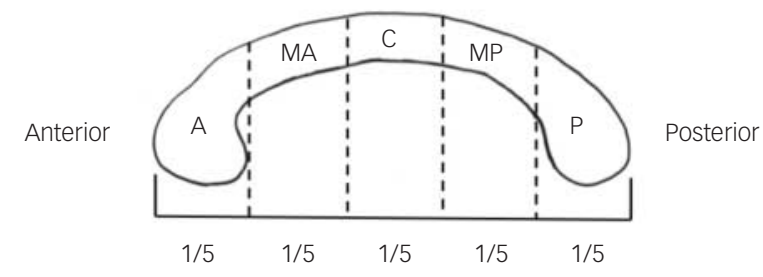

Fig. 1 Schematic of corpus callosum segmentation showing five equal segments.

$\mathrm{P}$, posterior (which is roughly equivalent of the posterior splenium); MP, mid-posterior (anterior splenium); C, central (truncus/body); MA, mid-anterior (genu); A, anterior (rostrum). 
patients and 73 controls (Table 1) were submitted for analysis following removal of extreme outliers (mean volume, area or fractional anisotropy greater than/less than 2 standard deviations from group mean). Of these patients, 65 had first-episode schizophrenia (first-episode group), with a mean duration of psychiatric illness of 2.31 years (s.d. $=2.46$ ). The remaining 48 participants with chronic schizophrenia (chronic group) had a significantly longer mean duration of psychiatric illness in comparison with the first-episode group $(P<0.001)$. The two patient subgroups did not differ significantly with respect to age at onset and the duration of untreated psychosis but there was a significant difference in mean daily chlorpromazine equivalent doses $(P<0.01)$. There were no significant differences in PANSS total or subscale scores or GAF scores between the first-episode and chronic groups. Overall, the patients had received marginally less education than healthy controls $(P<0.001)$.

\section{Corpus callosum midsagittal area comparisons}

Repeated measures ANCOVA of corpus callosum area revealed a significant main effect of diagnosis $(F(2,176)=6.58, P<0.05)$ between the chronic group and controls $(P=0.001)$ and a trend between the first-episode group and controls $(P=0.07)$. As shown in Table 2, corpus callosum midsagittal areas were consistently smaller in patients than in healthy controls. Post hoc analyses (Table 2) showed smaller areas in the first-episode group compared with controls in the mid-anterior corpus callosum and posterior corpus callosum. The chronic group demonstrated significantly smaller areas than controls in the mid-anterior corpus callosum, central corpus callosum, mid-posterior corpus callosum but no difference in posterior corpus callosum. Neither of the patient groups demonstrated any difference to controls in anterior corpus callosum area. First-episode and chronic groups showed a significant difference in mid-anterior corpus callosum only. Midsagittal areas were smallest in the chronic group in all corpus callosum regions and largest in healthy controls in all corpus callosum regions. No gender effects were observed in either the two patient groups or controls.

\section{Corpus callosum volume comparisons}

Repeated measures ANCOVA of corpus callosum volume revealed a significant main effect of diagnosis $(F(2,176)=7.03, P<0.05)$ between the chronic group and controls $(P=0.001)$ and a trend between the first-episode group and controls $(P=0.08)$. In the chronic group compared with controls, corpus callosum volumes were smaller in the mid-anterior, central and mid-posterior segments. Corpus callosum volumes were smaller in the firstepisode group than in healthy controls in the mid-anterior corpus callosum, central corpus callosum and posterior corpus callosum. Overall, the smallest volumes were recorded in the chronic group except posterior corpus callosum volume, whereas the largest volumes were found in the healthy individuals (Table 2). No gender effects in volume were observed in either of the two patient groups or controls.

\section{Fractional anisotropy comparisons}

The ANCOVA of fractional anisotropy showed no significant main effect for gender, diagnosis, or gender and diagnosis interaction in any corpus callosum region. No gender effects in DTI were observed in either the patient groups or controls (Table 3).

\section{Correlations}

In the patients, examined as a group or according to chronicity, there were no significant correlations between the number of years of education, the duration of psychiatric illness and duration of untreated psychosis with corpus callosum volume or DTI measures. Duration of psychiatric illness was negatively associated with central area $(r(113)=-0.18, P<0.05)$. A number of small but significant negative correlations were observed between daily antipsychotic dose and corpus callosum volume including whole corpus callosum volume $(r(113)=-0.17, P<0.05)$ as well as mid-anterior $(r(113)=-0.26, P<0.001)$, central $(r(113)=$ $-0.23, P<0.001)$ and mid-posterior volumes $(r(113)=-0.22$, $P<0.001)$ that were mirrored in area measures of total corpus callosum area $(r(113)=-0.19, P<0.05)$, mid-anterior $(r(113)=$ $-0.26, P<0.001)$, central $(r(113)=-0.23, P=0.001)$ and midposterior $(r(113)=-0.19, P<0.01)$ area. No DTI measures were correlated with antipsychotic dose.

Several correlations were performed between the corpus callosum areas and volumes in the control group to determine whether there was any natural association between the various measures used in the present investigations. Total midsagittal area of the corpus callosum was strongly correlated with total corpus callosum volume $(r(73)=0.78, P<0.001)$. Midsagittal areas of the anterior corpus callosum $(r=0.84)$, mid-anterior corpus

\begin{tabular}{|c|c|c|c|c|}
\hline & $\begin{array}{l}\text { First-episode group } \\
\qquad(n=65)\end{array}$ & $\begin{array}{l}\text { Chronic group } \\
\qquad(n=48)\end{array}$ & $\begin{array}{l}\text { Controls } \\
(n=73)\end{array}$ & $P$ \\
\hline Males, $n(\%)$ & $50(70.76)$ & $34(70.83)$ & $47(64.38)$ & ns \\
\hline Age, years: mean (s.d.) & $29.31(7.03)$ & $37.00(9.01)$ & $32.24(10.17)$ & $<0.001^{\mathrm{a}}$ \\
\hline Years of education, mean (s.d.) & $11.38(2.51)$ & $11.50(2.05)$ & $13.86(2.04)$ & $<0.001^{\mathrm{b}}$ \\
\hline Age at onset, years: mean (s.d.) & $26.31(7.07)$ & $24.23(6.76)$ & - & $\mathrm{ns}$ \\
\hline Duration of illness, years: mean (s.d.) & $2.31(2.46)$ & $12.04(8.50)$ & - & $<0.001$ \\
\hline Duration of untreated psychosis, years: mean (s.d.) & $1.55(2.09)$ & $1.18(1.22)$ & - & ns \\
\hline \multicolumn{5}{|l|}{ Positive and Negative Syndrome Scale, mean (s.d.) } \\
\hline Positive & $10.73(3.95)$ & $10.58(3.88)$ & - & ns \\
\hline Negative & $9.06(3.22)$ & $8.83(3.16)$ & - & ns \\
\hline General psychopathology & $20.98(4.05)$ & $19.66(2.93)$ & - & ns \\
\hline Total & $40.78(8.91)$ & $39.08(8.03)$ & - & ns \\
\hline Global Assessment of Functioning, total: mean (s.d.) & $52.91(19.30)$ & $51.11(16.37)$ & - & ns \\
\hline Daily chlorpromazine equivalent dose, mg: mean (s.d.) & $156.15(141.35)$ & $239.58(200.12)$ & - & ns \\
\hline
\end{tabular}


Table 2 Mean midsagittal corpus callosum regional areas $\left(\mathrm{mm}^{2}\right)$ and volumes $\left(\mathrm{mm}^{3}\right)$ in the first-episode and chronic patient groups and the controls

\begin{tabular}{|c|c|c|c|c|c|c|}
\hline \multirow[b]{2}{*}{ Corpus callosum region } & \multicolumn{3}{|c|}{ Mean (s.d.) } & \multicolumn{3}{|c|}{ Post hoc comparisons, $P$} \\
\hline & $\begin{array}{l}\text { Chronic group } \\
\qquad(n=48)\end{array}$ & $\begin{array}{l}\text { First-episode group } \\
\qquad(n=65)\end{array}$ & $\begin{array}{l}\text { Controls } \\
(n=73)\end{array}$ & $\begin{array}{l}\text { First-episode group } \\
\text { v. chronic group }\end{array}$ & $\begin{array}{l}\text { First-episode group } \\
\text { v. controls }\end{array}$ & $\begin{array}{l}\text { Chronic group } \\
\text { v. controls }\end{array}$ \\
\hline \multicolumn{7}{|l|}{ Area } \\
\hline Anterior & $166.56(33.16)$ & 170.00 (33.84) & $172.67(25.96)$ & ns & ns & ns \\
\hline Mid-anterior & 93.68 (17.35) & $108.32(28.51)$ & $125.12(40.36)$ & $<0.05$ & $<0.01$ & $<0.001$ \\
\hline Central & $91.35(23.70)$ & $111.16(43.58)$ & $128.63(51.94)$ & 0.05 & 0.05 & $<0.001$ \\
\hline Mid-posterior & $86.29(19.50)$ & 93.50 (19.65) & $102.02(26.53)$ & ns & ns & 0.001 \\
\hline Posterior & 182.73 (32.68) & $185.25(24.63)$ & $188.12(29.15)$ & ns & $<0.05$ & ns \\
\hline Whole & 623.14 (79.95) & $665.73(108.86)$ & $723.27(132.40)$ & ns & $<0.01$ & $<0.001$ \\
\hline \multicolumn{7}{|l|}{ Volume } \\
\hline Anterior & $822.60(137.85)$ & $841.63(157.35)$ & $862.10(140.56)$ & ns & ns & ns \\
\hline Mid-anterior & $445.24(87.86)$ & $496.52(116.20)$ & $568.67(143.83)$ & ns & $<0.01$ & $<0.001$ \\
\hline Central & $411.52(81.91)$ & $457.47(101.11)$ & $515.16(111.05)$ & 0.05 & $<0.01$ & $<0.001$ \\
\hline Mid-posterior & $407.87(86.28)$ & $448.46(87.21)$ & $478.67(91.96)$ & 0.05 & ns & $<0.001$ \\
\hline Posterior corpus callosum & 922.20 (123.25) & $916.26(158.87)$ & 977.65 (141.72) & ns & $<0.05$ & ns \\
\hline Whole corpus callosum & 3009.45 (384.71) & 3160.34 (471.90) & 3402.27 (480.09) & ns & $<0.01$ & $<0.001$ \\
\hline
\end{tabular}

Table 3 Mean corpus callosum fractional anisotropy values in patients and controls ${ }^{\mathrm{a}}$

\begin{tabular}{|lccc|} 
& \multicolumn{3}{c|}{ Mean (s.d.) } \\
\cline { 2 - 4 } Corpus callosum & $\begin{array}{c}\text { Chronic group } \\
(n=48)\end{array}$ & $\begin{array}{c}\text { First-episode } \\
\text { group }(n=65)\end{array}$ & $\begin{array}{c}\text { Controls } \\
(n=73)\end{array}$ \\
\hline Anterior & $0.493(0.08)$ & $0.492(0.08)$ & $0.513(0.08)$ \\
\hline Mid-anterior & $0.433(0.07)$ & $0.438(0.07)$ & $0.430(0.05)$ \\
\hline Central & $0.493(0.08)$ & $0.501(0.09)$ & $0.519(0.07)$ \\
\hline Mid-posterior & $0.526(0.10)$ & $0.541(0.09)$ & $0.536(0.08)$ \\
\hline Posterior & $0.667(0.12)$ & $0.652(0.12)$ & $0.672(0.09)$ \\
\hline a. All values rounded off to three significant figures. & \\
\hline
\end{tabular}

callosum $(r=0.77)$, central corpus callosum $(r=0.55)$, midposterior corpus callosum $(r=0.77)$ and posterior corpus callosum $(r=0.77)$ were all significantly correlated with volumes of the respective regions (all d.f. $=73, P<0.001$ ).

\section{Discussion}

To the best of our knowledge, this study examines the corpus callosum in one of the largest cohorts of patients with schizophrenia thus far and is the first study of its kind in an Asian population. The availability of patients with both first-episode and chronic schizophrenia provided a direct comparison between groups to better elucidate the effects of age, gender, age at onset and chronicity on corpus callosum integrity. In addition, both the midsagittal area and the volume of the corpus callosum and its subdivisions were examined thereby overcoming the problems associated with the selection and use of a single midsagittal slice. Lastly, as DTI fractional anisotropy values were also obtained from the participants, an investigation of the relationships between corpus callosum area, volume and fractional anisotropy could be performed.

\section{Callosal area and volume}

Corpus callosum size was significantly reduced in patients compared with controls in line with the majority of studies conducted thus far. ${ }^{16}$ The size difference observed in the mid-anterior segments of the corpus callosum bore a resemblance to those found by
Goghari et $a l,{ }^{17}$ as in our study smaller areas were detected in the rostral body and anterior midbody of the corpus callosum. Walterfang et $a l^{5}$ examined callosal morphology in a large sample of patients with first-episode and chronic schizophrenia and found reductions in callosal width in the anterior genu in those with first-episode schizophrenia with similar reductions in those whose condition was chronic in the anterior genu, but also in the posterior genu and isthmus, suggesting possible early anterior and late posterior change at the chronic phase of illness.

In line with Walterfang and colleagues' study, significant area reductions were apparent in the mid-anterior and posterior areas in the first-episode group, whereas a greater area reduction overall was found in the chronic group across the entire callosum - particularly in the mid-anterior section excluding the anterior segment and central corpus callosum. Additionally, negative correlations were seen between the duration of illness and corpus callosum area. Mitelman and colleagues ${ }^{18}$ have reported smaller corpus callosum size and anisotropy in patients with chronic schizophrenia compared with normal controls at baseline and after comparing baseline MRI scans with subsequent scans 4 years later. They also found that the corpus callosum at baseline was of a smaller size, more elongated and posteriorly positioned in patients with poor outcome compared with those with a good outcome. In healthy people, corpus callosum mid-anterior development appears to be ongoing into adulthood and, in schizophrenia, greater reductions could suggest a specific failure of myelination that occurs normally in childhood and adolescence. ${ }^{3,4,19,20}$

One key difference that sets the present study apart from previous studies is the use of corpus callosum volume for comparisons, instead of only corpus callosum area measurements calculated from the midsagittal slice. Midsagittal slice selection procedures vary considerably, and although the strong correlations between corpus callosum area measures and corpus callosum volume measures imply that studies employing the former and the latter can be compared, these measures may lead to different conclusions in abnormal states. Studies of area in early-stage schizophrenia indicate predominantly anterior and posterior changes, ${ }^{5,19,20}$ whereas volumetric measures in our study showed changes in central and posterior corpus callosum volume in the first-episode group that may suggest early evidence of a failure of the central and posterior corpus callosum to develop with illness onset. 
Previous studies also show anterior and posterior corpus callosum area changes in patients with chronic schizophrenia, ${ }^{3,4,17}$ whereas our data showed corpus callosum volume reductions were more prominent throughout mid-anterior to mid-posterior subsections of those in the chronic group. Furthermore, when examining volume, we found more central changes that also correlated with duration of illness, which is in line with the view that the central corpus callosum is changing dynamically with age and may be at the intersection of age-related and disease-affected developmental trajectories. Despite reasonable correlations between area and volume in healthy controls, in our patients the present findings suggest that the extent of size difference captured in the midsagittal slice is unlikely to be equivalent to the degree of structural abnormality outside the selected slice and, as such, white matter anomalies may not be reflected accurately when corpus callosum midsagittal areas were compared.

\section{Absence of gender differences}

The absence of gender differences in the corpus callosum measures in controls is a notable aspect of the study. In the general healthy population the majority of MRI studies have reported larger corpus callosums in females, although studies with the opposite finding also appear in the literature. In a meta-analysis of 43 MRI studies investigating the effects of gender, age and handedness on corpus callosum morphology, Driesen \& $\mathrm{Raz}^{21}$ found that absolute corpus callosum area and area of the splenium was greater in males than females but when overall brain size or intracranial volume is controlled for the direction of the gender, difference was reversed. Notably, Jancke et $a l^{22}$ reported that the only apparent influence of gender was on corpus callosum ratios, which were larger in females. However, smaller brains had larger corpus callosum ratios that were mainly independent of gender. In line with this, our results indicated no gender differences in controls' corpus callosum measures when whole brain volume and age are considered. Whether this difference is also partially attributable to generally larger corpus callosum ratios in Asian people remains to be determined by cross-cultural studies but presently little data exists that can clarify the relationship of corpus callosum to brain volume in this population. Takeda et $a l^{23}$ in a healthy Japanese sample, showed that although the widths of rostrum, body and splenium of the corpus callosum became thinner with age and the anterior to posterior length and height of the corpus callosum increased with age, no gender differences in regional size of corpus callosum were observed.

Consistent with the majority of studies, our findings showed no significant effect of gender in schizophrenia, suggesting that, in schizophrenia, similarities in morphology between them outweigh the differences. ${ }^{24}$ Some post-mortem investigations have shown callosum abnormalities that interact with gender ${ }^{25}$ but our data agree with the weight of imaging evidence suggesting no significant gender disparity in callosal change. It is possible that the older corpus callosums of post-mortem investigations are not comparable with younger ones of MRI investigations because of age-related differences in myelination and/or the familiar problems of participant heterogeneity, failure to correct for multiple comparisons, failure to control for total brain size and/or poor age matching between groups.

\section{Fractional anisotropy}

Despite observing a significant decrease in corpus callosum area and volume in patients relative to healthy controls, no significant fractional anisotropy differences were found. This finding is more unusual in patients with chronic schizophrenia but not unprecedented in patients with a first episode. Price et $a l^{26}$ reported negative findings in 20 patients with first-episode schizophrenia using a region of interest approach in the splenium and genu of the corpus callosum. More recently, Kong and colleagues, ${ }^{27}$ using both voxel-based and fibre-tracking DTI in a small Chinese sample, reported significantly decreased fractional anisotropy values in the genu in patients with chronic but not first-episode schizophrenia. Indeed, disturbances in white matter integrity in general appear to be less consistent in patients with first-episode than in those with chronic schizophrenia, ${ }^{28}$ suggesting that in the early stages of schizophrenia there may be little or no disruption to the functional integrity of the corpus callosum at least in some patients. As such, sampling differences in duration of illness and length of illness may be important factors in determining whether fractional anisotropy changes. As noted by Walterfang et al, ${ }^{29}$ 'One of the difficulties in neuroimaging research into schizophrenia remains the heterogeneity of the illness, and subtle changes may be lost when individuals affected to different degrees and by potentially differing underpinning neurobiology are pooled' (p. 935). Finally, it is worth noting that whereas reduced corpus callosum size suggests a loss of interhemispheric axons, changes in anisotropy do not necessarily follow a reduction in the number of axons. ${ }^{30,31}$

\section{Study limitations and future directions}

Apart from the obvious limitations of cross-sectional studies, several other limitations should be acknowledged. In particular, a drawback of the fractional anisotropy automated method lies in the arbitrary delineation of the lateral boundary of the corpus callosum and callosal fibres are contiguous with white matter, which makes it impossible to precisely identify the entire corpus callosum in its lateral aspects. A further negative of automatic parcellation methods is that the five subregions obtained do not correspond exactly with distinct anatomical or functional corpus callosum subregions in reality. On the positive side, the same method was applied for all participants and can be compared in subsequent studies that employ FreeSurfer. Future studies may benefit from adding diffusion tensor tractography to assist in corpus callosum parcellation, as the technique allows the mapping of specific regions in the corpus callosum and their continuity into connected cortical regions.

Another important limitation is the unknown effect of lifetime antipsychotic medication. On the one hand, negative correlations have been reported between corpus callosum area and estimated lifetime antipsychotic consumption ${ }^{3}$ and conversely there is also evidence that psychotropic medications may prevent neuronal white matter loss or protect against the pathophysiological effect of the disease process via the simulation of glial cell genesis, which creates myelin. ${ }^{32}$ Although we were not able to measure lifetime antipsychotic exposure, covarying for the effect of daily antipsychotic medication did not reveal any new insights or alter the pattern of findings. Future studies would benefit from considering the influence of long-term exposure to antipsychotics.

A final limitation of the current study lies in the small number of left-handed participants, thereby ruling out an investigation of the effects of handedness on corpus callosum size and anisotropy. Nevertheless, handedness probably has a minimal impact in this study, since the removal of all left-handed and ambidextrous participants from the analyses did not alter results.

In conclusion, reductions in the size of the corpus callosum appears to be a consistent feature of schizophrenia but do not appear to differ between the genders, at least in Chinese Singaporean patients. Age-related decline in the corpus callosum may be less apparent or yet to occur fully in patients with first-episode 
schizophrenia but is exacerbated by more chronic illness, whereas differences in connectivity may vary with illness heterogeneity. These findings point to individual variations in the developmental trajectory of callosum growth and abnormality. Further studies are required to further elucidate how individual differences play out in corpus callosum function across the illness spectrum.

Simon L. Collinson, DPhil, Department of Psychology, National University of Singapore and Research Division, Woodbridge Hospital/Institute of Mental Health, Singapore; Swu Chyi Gan, MSocSci, Department of Psychology, National University of Singapore, Singapore; Puay San Woon, BSC, Carissa Kuswanto, MSC, Min Yi Sum, BA, Research Division, Woodbridge Hospital/Institute of Mental Health, Singapore; Guo Liang Yang, PhD, Ji Min Lui, PhD, Biomedical Imaging Laboratory, Singapore Bioimaging Consortium, Agency for Science, Technology and Research, Singapore; Yih Yian Sitoh, Department of Neuroradiology, National Neuroscience Institute, Singapore; Wieslaw L. Nowinski, DSC, PhD, Biomedical Imaging Laboratory, Singapore Bioimaging Consortium, Agency for Science, Technology and Research, Singapore; Kang Sim, MBBS, MMed, FAMS, Research Division and Department of General Psychiatry, Woodbridge Hospital/Institute of Mental Health, Singapore

Correspondence: Simon L. Collinson, Department of Psychology, National University of Singapore, Block AS4, Unit \#02-07, 9 Arts Link, Singapore 117570. Email psycs|@nus.edu.sg

First received 12 Feb 2013, final revision 26 Jun 2013, accepted 5 Sep 2013

\section{Funding}

Supported by the National Healthcare Group Research Grant (NHG-SIG/05004) an Singapore Bioimaging Consortium Research Grant (SBIC RP C-009/2006) and Nationa University of Singapore FASS Staff Research Support Grant.

\section{References}

1 Arnone D, McIntosh AM, Tan GMY, Ebmeier KP. Meta-analysis of magnetic resonance imaging studies of the corpus callosum in schizophrenia. Schizophr Res 2008; 101: 124-32.

2 John PJ, Shakeel MK, Jain S. Corpus callosal area differences and gende dimorphism in neuroleptic-naïve, recent-onset schizophrenia and healthy control subjects. Schizophr Res 2008; 103: 11-21.

3 Bersani G, Quartini A, lannitelli A, Paolemili M, Ratti F, Di Biasi C, et al. Corpus callosum abnormalities and potential age effect in men with schizophrenia: an MRI comparative study. Psychiatry Res 2010; 183: 119-25.

4 Downhill JE, Buchsbaum MS, Wei T, Spiegel-Cohen J, Hazlett EA, Haznedar MM, et al. Shape and size of the corpus callosum in schizophrenia and schizotypal personality disorder. Schizophr Res 2000; 42: 193-208.

5 Walterfang M, Wood AG, Reutens DC, Wood SJ, Chen J, Velakoulis D, et al. Morphology of the corpus callosum at different stages of schizophrenia: cross-sectional study in first-episode and chronic illness. Br J Psychiatry 2008; 192: 429-34.

6 Keller A, Jeffries NO, Blumenthal J, Clasen LS, Liu H, Giedd JN, et al. Corpus callosum development in childhood-onset schizophrenia. Schizophr Res 2003; 62: 105-14.

7 Walterfang M, Wood AG, Reutens DC, Phillips L, Wood SJ, Chen J, et al. Corpus callosum shape alterations in individuals prior to the onset of psychosis. Schizophr Res 2008; 103: 1-10.

8 American Psychiatric Association. Diagnostic and Statistical Manual of Mental Disorders (4th edn) (DSM-IV). APA, 1994.

9 First MB, Spitzer RL, Gibbon M, Williams JBW. Structured Clinical Interview for DSM-IV Axis I Disorders-Patient Version (SCID-P). American Psychiatric Press, 1994

10 First MB, Spitzer RL, Gibbon M, Williams JBW. Structured Clinical Interview for DSM-IV Axis I Disorders-Non-patient Edition (SCID-NP). American Psychiatric Press, 2002.
11 Kay SR, Fiszbein A, Opler LA. The positive and negative syndrome scale (PANSS) for schizophrenia. Schizophr Bull 1987; 13: 261-76.

12 Schachter SC, Ransil BJ, Geschwind N. Associations of handedness with hair color and learning disabilities. Neuropsychologia 1987; 25: 269-76.

13 Nowinski WL, Prakash B, Volkau I, Ananthasubramaniam A, Beauchamp Jr NJ. Rapid and automatic calculation of the midsagittal plane in magnetic resonance diffusion and perfusion images. Acad Radiol 2006; 13: 652-63.

14 Fischl B, Salat DH, Busa E, Albert M, Dieterich M, Haselgrove C, et al. Whole brain segmentation: automated labeling of neuroanatomical structures in the human brain. Neuron 2002; 33: 341-55.

15 Jiang $\mathrm{H}$, van Zijl PC, Kim J, Pearlson GD, Mori S. Dtistudio: resource program for diffusion tensor computation and fiber bundle tracking. Comput Methods Programs Biomed 2006: 81: 106-16.

16 Woodruff PWR, McManus IC, David AS. Meta-analysis of corpus-callosum size in schizophrenia. J Neurol Neurosurg Psychiatr 1995; 58: 457-61.

17 Goghari VM, Lang DJ, Flynn SW, MacKay AL, Honer WG. Smaller corpus callosum subregions containing motor fibres in schizophrenia. Schizophr Res 2005; 73: 59-68

18 Mitelman SA, Nikiforova YK, Canfield EL, Hazlett EA, Brickman AM, Shihabuddin L, et al. A longitudinal study of the corpus callosum in chronic schizophrenia. Schizophr Res 2009; 114: 144-53.

19 Bachmann S, Pantel J, Flender A, Bottmer C, Essig M, Schröder J. Corpus callosum in first-episode patients with schizophrenia - a magnetic resonance imaging study. Psychol Med 2003; 33: 1019-27.

20 Keshavan MS, Diwadkar VA, Harenski K, Rosenberg DR, Sweeney JA, Pettegrew JW. Abnormalities of the corpus callosum in first-episode treatment naïve schizophrenia. J Neurol Neurosurg Psychiatry 2002; 72 : $757-60$.

21 Driesen NR, Raz N. The influence of sex, age, and handedness on corpus callosum morphology: a meta-analysis. Psychobiology 1995; 23: 240-7.

22 Jancke L, Staiger JF, Schlaug G, Huang Y, Steinmetz H. The relationship between corpus callosum size and forebrain volume. Cereb Cortex 1997; 7: $48-56$.

23 Takeda $\mathrm{S}$, Hirashima $\mathrm{Y}$, Ikeda $\mathrm{H}$, Yamamoto $\mathrm{H}$, Sugino $\mathrm{M}$, Endo $\mathrm{S}$. Determination of indices of the corpus callosum associated with normal aging in Japanese individuals. Neuroradiol 2003; 45: 513-8.

24 Lewis S. Sex and schizophrenia: vive la différence. Br J Psychiatry 1992; 161: $445-50$.

25 Highley JR, Esiri MM, McDonald B, Cortina-Borja M, Herron BM, Crow TJ. The size and fibre composition of the corpus callosum with respect to gender and schizophrenia: a post-mortem study. Brain 1999; 122: 99-110.

26 Price G, Bagary MS, Cercignani M, Altmann DR, Ron MA. The corpus callosum in first episode schizophrenia: a diffusion tensor imaging study. J Neurol Neurosurg Psychiatry 2005; 76: 585-7.

27 Kong X, Ouyang X, Tao H, Liu H, Li L, Zhao J, et al. Complementary diffusion tensor imaging study of the corpus callosum in patients with first-episode and chronic schizophrenia. J Psychiatry Neurosci 2011; 36: 120-5.

28 Fitzsimmons J, Kubicki M, Shenton MW. Review of functional and anatomical brain connectivity findings in schizophrenia. Curr Opin Psychiatr 2013; 26: $172-87$.

29 Walterfang M, Wood SJ, Velakoulis D, Pantelis C. Neuropathological, neurogenetic and neuroimaging evidence for white matter pathology in schizophrenia. Neurosci Biobehav Rev 2006; 30: 918-48.

30 Cercignani M, Horsfield MA. The physical basis of diffusion-tensor weighted MRI. J Neurol Sci 2001; 186: S11-4.

31 Hugenschmidt CE, Peiffer AM, Kraft RA, Casanova R, Deibler AR, Burdette JH, et al. Relating imaging indices of white matter integrity and volume in healthy older adults. Cereb Cortex 2007; 18: 433-42.

32 Bartzokis G, Lu PH, Neuchterlein KH, Gitlin M, Doi C, Edwards N, et al. Differential effects of typical and atypical antipsychotics on brain myelination in schizophrenia. Schizophr Res 2007; 93: 13-22. 